\title{
COMPETÊNCIAS E O PROCESSO ENSINO-APRENDIZAGEM DO DIAGNÓSTICO DE ENFERMAGEM*
}

\section{Resumo}

Trata-se de um estudo qualitativo, em nível exploratório, descritivo e interpretativo, e teve como objetivo relacionar as concepções que discentes e docentes possuem sobre competências com o processo ensinoaprendizagem do diagnóstico de enfermagem. As informações foram coletadas em duas etapas, primeiramente através de oito entrevistas individuais semi-estruturadas. Num segundo momento, foram realizados dois grupos focais, um de docentes e outro de discentes. Utilizou-se Análise de Conteúdo como metodologia de análise. Como resultado emergiram três categorias: Relação com os saberes/conhecimentos, Estratégias de ensinoaprendizagem e Contexto Institucional do Curso.

Descritores: competência profissional; diagnóstico de enfermagem; ensino; aprendizagem

\begin{abstract}
This is a qualitative, exploratory, descriptive and interpretative study. The objective of the study is to relate the conceptions that professors and students have over competencies with the teaching-learning process of nursing diagnosis. The study was carried out at the Nursing School of the Federal University of Rio Grande do Sul. The information was collected in two stages. The first one through eight semi-structured individual interviews and the second one through two focus groups. One of them was formed by students and the other group was formed by professors. Content analysis was used as analysis methodology. As a result three categories were found: relation with knowledge, teaching-learning strategies and institutional context of the course.

Descriptors: professional competence; nursing diagnosis; teaching; learning Title: Competency for teaching and learning nursing diagnosis
\end{abstract}

\section{Resumen}

Este estudio es de cuño cualitativo, en nivel exploratorio, descriptivo, interpretativo, y tiene como objectivo relacionar las concepciones de alumnos y docentes sobre las competencias con el processo de enseñanza del diagnostico de enfermeria. La investigación se realizó en la Escuela de Enfermeria de la Universidad Federal del Rio Grande del Sur. Las informaciónes fuerón colectadas en dos etapas, primeramente a través de ocho entrevistas individuales semiestructuradas. En un segundo momento fuerón realizados dos grupos focales, uno con docentes y otro con los alumnos. Utilizé análisis de contenido como metodologia de análisis. Como resultado emergierón tres categorias: relación con el saber/conocimiento, estrategias de enseñanza y contexto institucional del curso .

Descriptores: competencia profesional; diagnostico de enfermería; enseñanza; aprendizaje

Título: Competencias y el processo de enseñanza del diagnostico de enfermeria

\section{Introdução}

No presente estudo tenciono contribuir com o debate acerca de dois temas, ligados à educação e à enfermagem, cujos estudos multiplicam-se no momento em que o terreno está sendo ampliado. Falar do desenvolvimento de competências no ensino de enfermagem, na ocasião em que as Diretrizes Curriculares Nacionais para o Curso de Graduação em Enfermagem as utilizam como concepção nuclear orientadora, e os Projetos Pedagógicos de cada curso estão em fase de elaboração ou implantação, consiste num grande desafio. Além disso, incluir neste estudo os diagnósticos de enfermagem, tema ainda tão polêmico entre os profissionais da área, parece-me, à primeira vista, uma conexão questionável e até mesmo temerária. Porém, a argumentação que advogo é de que o ensino e a aprendizagem do diagnóstico de enfermagem contribuem para o desenvolvimento da competência das futuras enfermeiras, além de requerer o desenvolvimento de outras competências mais específicas para a sua realização.

Em todas as áreas de atuação, o homem desenvolve suas atividades de forma organizada, seguindo um método para atingir seus propósitos. Na prática profissional da enfermeira (os termos enfermeiro e enfermeira, professor e professora, aluno e aluna serão empregados aleatoriamente neste estudo, tendo em vista que a sociedade é composta por homens e mulheres), a metodologia empregada na organização do conhecimento e do cuidado ao paciente ${ }^{a}$ denomina-se Processo de Enfermagem (PE). O seu uso possibilita a aplicação dos fundamentos teóricos da enfermagem na prática, ordenando e direcionando o cuidado de forma individualizada, personalizada e humanizada.
O PE constitui uma atividade intelectual deliberada que auxilia a enfermeira na tomada de decisões, cujo foco reside na obtenção dos resultados esperados. Atualmente, este método é desenvolvido em cinco etapas inter-relacionadas e interdependentes, caracterizando-se por ser intencional, sistemático, dinâmico, interativo, flexível e baseado em teorias.

O ensino do PE em nosso meio teve seu início com Horta $^{(1)}$, na década de 70 . Ele fornecia uma estrutura de pensamento para que a enfermeira antecipasse ou resolvesse rapidamente os problemas apresentados pelos pacientes. A sua aplicação, contudo, se dava de forma incompleta. Uma importante lacuna consistia no estabelecimento dos problemas do paciente, ou problemas de enfermagem, como era denominada a etapa que hoje se chama diagnóstico de enfermagem. Não havia consenso sobre o que deveria ser registrado, embora soubéssemos prescrever cuidados concernentes à equipe de enfermagem.

$\mathrm{Na}$ década de 70, um grupo de enfermeiras norteamericanas e canadenses reuniu-se com o objetivo de construir uma terminologia de caráter internacional, que referisse os problemas de saúde do cliente pelos quais tem responsabilidade profissional. O trabalho deste grupo deu origem à taxonomia da North American Nursing Diagnosis Association (NANDA), que consiste num sistema de classificação dos diagnósticos de enfermagem. Esta padronização da nomenclatura é necessária, também, para a informatização dos registros das enfermeiras e para respaldar legalmente suas ações, reforçando a autonomia profissional.

O termo Diagnóstico de Enfermagem (DE) tem sido compreendido tanto como um processo quanto como um

\footnotetext{
* Texto construído a partir de: Almeida MA. Competências e o processo ensino-aprendizagem do diagnóstico de enfermagem: concepções de docentes e discentes. Porto Alegre (RS): Faculdade de Educação, PUC-RS; 2002. 245f. **Enfermeira. Professora Adjunto da Escola de Enfermagem da Universidade Federal do Rio Grande do Sul. Doutora em Educação/PUCRS.

E-mail do autor: miriam.abreu@terra.com.br

a $\mathrm{O}$ termo paciente aqui empregado pode ser substituído por indivíduo, família, grupo ou comunidade, ou seja, aqueles de quem a enfermagem cuida.
} 
produto. O processo diagnóstico pode ser considerado um subprocesso do PE, antecedendo o enunciado desse produto, momento em que é empregada uma nomenclatura padronizada. Entender os processos e produtos do raciocínio diagnóstico, influenciados por teorias e conceitos do processamento da informação e tomada de decisão se tornou o foco do ensino clínico em muitos países. No Brasil, o ensino e a pesquisa dos DE tiveram seu início no final da década de 80, decorrentes do intercâmbio técnico-científico-cultural entre docentes da Universidade Federal da Paraíba e da Universidade de Connecticut - EUA(2). A adoção de livros textos, majoritariamente traduções de obras norte-americanas que utilizam a NANDA, impulsionou o estudo da taxonomia. Contudo, ainda hoje se observa que essa etapa é ensinada de forma isolada e descontínua nos cursos de graduação.

Poucas instituições brasileiras aplicam o DE na prática profissional. Aliado à falta de dados que permitam a sua determinação, atribui-se a esse fato o desconhecimento e a dificuldade de domínio técnico-científico da enfermeira na identificação das respostas humanas do paciente diante de uma situação de estresse. Somada a isso, identifica-se a resistência de profissionais em empregar a metodologia do $P E$, por entendê-la como uma atividade burocrática que os distancia do paciente.

Imagens simplificadas e ilusórias em relação ao Processo de Enfermagem, tanto ao considerá-lo como algo difícil de ser concretizado e meramente acadêmico, quanto ser a única alternativa para o enfermeiro impede que se reconheça sua real atividade ${ }^{(3,4)}$. Esta é uma discussão que deve permear o ensino e o contexto dos serviços. As vantagens e desvantagens do PE continuam a ser debatidas na literatura.

As associações existentes em diferentes países atestam o atual estado da arte na área da nomenclatura, conceitos e desenvolvimento de sistemas de classificação em enfermagem, decorrentes da demanda crescente por cuidados de enfermagem requeridos pelas mudanças sociais, políticas, tecnológicas e culturais. Além disso, as enfermeiras das diversas nações têm sido solicitadas a divulgar seu trabalho e a demonstrar a eficiência e eficácia dos cuidados de enfermagem dispensados à clientela. Nesta perspectiva, é visualizada a importância do desenvolvimento de uma terminologia que expresse a prática profissional alicerçada em sólido conhecimento.

A disseminação deste conhecimento que está sendo produzido se dá, especialmente, via educação. É função da Universidade, dentre outras, a difusão do saber com consciência crítica e pensamento criador $^{(5)}$. A utilização das classificações de diagnósticos, intervenções e resultados de enfermagem demanda julgamento clínico e tomada de decisão, que precisam ser ensinados e aprendidos nos cursos de formação.

O DE representa uma mudança de paradigma de uma profissão voltada para aspectos biomédicos, preocupada com o processo patológico e essencialmente dependente, para uma profissão que busca sua função independente, ao focalizar as respostas fisiológicas, psicológicas, sociais, culturais e espirituais do indivíduo, família e comunidade que dêem respaldo ao cuidado de enfermagem.

A compreensão do processo de raciocínio diagnóstico favorece não apenas a própria enfermeira, mas a sociedade, que passa a desfrutar de cuidados mais apropriados e qualificados decorrentes da identificação mais segura e precoce dos problemas do paciente(2). Esta visão, no meu entender, não pode prescindir da participação do próprio sujeito que é cuidado.

A enfermeira deve ser competente na sua atividade diagnóstica, pois os diagnósticos de enfermagem expressam as necessidades de cuidados daqueles dos quais cuida. E a competência no exercício profissional implica ser competente para decidir os cuidados necessários ${ }^{(6)}$.

Assim, o ensino e a aprendizagem do processo diagnóstico assumem vital importância na formação dos futuros enfermeiros, cuja preocupação central e final é o indivíduo. Preocupa-me, porém, que a introdução dos diagnósticos de enfermagem no ensino e na assistência, informatizados ou não, concentre nossas atenções em normas preestabelecidas que configurem uma camisa-de-força, ou que o fascínio tecnológico suplante $o$ ato de pensar ou mesmo mascare a permanência no paradigma alicerçado na doença. Ou, ainda, que apresente um efeito rebote, distanciando a enfermeira do paciente.

O momento atual, em que a discussão sobre o ensino dos diagnósticos de enfermagem se intensifica, concorre com o indispensável debate nas Escolas e Faculdades do país acerca das Diretrizes Curriculares para o Curso de Graduação em Enfermagem ${ }^{(7)}$. Com a promulgação da LDB de 1996, as diretrizes curriculares vieram substituir os currículos mínimos até então existentes, configurando orientações gerais a serem observadas por todas as instituições de ensino superior na reformulação dos seus projetos pedagógicos.

A pedagogia de competências, contida nas diretrizes curriculares, encerra um paradigma possivelmente desconhecido por muitos daqueles envolvidos diretamente na práxis educativa. O centro das contradições da escola oscila entre dois paradigmas, o de ensinar conhecimentos ou desenvolver competências. O primeiro deles compreende uma abordagem tradicional que privilegia aulas e provas, enquanto o segundo fundamenta-se especialmente nas novas pedagogias e na formação de adultos ${ }^{(8,9)}$.

A expressão competência tem sido, por vezes, empregada na literatura e nos discursos vigentes como sendo um conceito atual, unívoco, politicamente neutro e consensual. No entando, é um termo polissêmico e tem sido apoiado nos mais diversos enfoques e paradigmas teóricos ${ }^{(8,10-13)}$.

O termo competência, mais recentemente utilizado no plural, invadiu não apenas o mundo do trabalho como o campo da educação, em seus diferentes níveis. Contudo, não se trata de várias competências, mas de componentes articulados de uma mesma competência(13). Há competências gerais e específicas que derivam da competência profissional, configurando desdobramentos ou detalhamentos deste todo da pessoa. Competência é definida como "uma capacidade de agir eficazmente em um determinado tipo de situação, apoiado em conhecimentos, mas sem limitar-se a eles"(8:7).

Pressupondo que:

a competência profissional compreende uma totalidade, que pode ser entendida como possuindo variadas dimensões;

o processo de enfermagem utilizado pelas enfermeiras na prática profissional possibilita cuidar do ser humano na sua integralidade, sendo que o diagnóstico de enfermagem, segunda etapa desta metodologia, propicia a base científica para as ações independentes;

a construção do diagnóstico, que implica processo interpretativo e complexo de julgamento e raciocínio clínico, possibilita identificar as respostas fisiológicas, psicológicas, sociais, culturais e espirituais do indivíduo, família e comunidade diante de processos vitais ou problemas de saúde, que dêem respaldo a uma ação assistencial concreta e contextualizada, de forma adequada e satisfatória;

Defendo que o ensino e a aprendizagem do diagnóstico de enfermagem, numa perspectiva integralizadora, ao envolver habilidades de pensamento crítico e de tomada de decisão, favorecem a construção da competência do formando em enfermagem e requerem o desenvolvimento de uma série de competências específicas e habilidades, inerentes ao ofício docente e ao discente. Desta forma, o diagnosticar em enfermagem abarca, a priori, dimensões de competência técnico-científica, ética, cultural, social, espiritual e em tecnologias da informação e comunicação, a serem contempladas na formação da enfermeira ao longo do curso. 
Partindo do anteriormente exposto, formulou-se o seguinte objetivo: relacionar as concepções que discentes e docentes possuem sobre competência com o processo ensinoaprendizagem do diagnóstico de enfermagem.

\section{Metodologia}

Trata-se de uma pesquisa qualitativa de nível exploratório, descritivo e interpretativo. As informações foram coletadas por meio de entrevistas individuais e de grupo focais ${ }^{(14-16)}$, empregados seqüencialmente. Na primeira etapa foram realizadas oito entrevistas individuais semi-estruturadas, cuja categorização dos resultados foram utilizados como temas propulsores nos grupos focais. Num segundo momento foram formados dois grupos focais, um de docentes e outro de discentes, cada um com quatro sessões para coleta de informações e uma para validação dos resultados. Utilizei Análise de Conteúdo ${ }^{(17)}$ como metodologia de análise. O estudo foi realizado na Escola de Enfermagem da Universidade Federal do Rio Grande do Sul (UFRGS) cujo hospital universitário introduziu o diagnóstico de enfermagem informatizado(18), na sistematização da assistência aos pacientes internados em seus 725 leitos, de modo que a sua aplicação extrapola o uso meramente acadêmico, muitas vezes dissociado da prática. Participaram do estudo onze discentes dos dois últimos semestres do curso de graduação e dez docentes que acompanhavam os alunos em campo de estágio, e que estavam utilizando os diagnósticos de enfermagem informatizados.

\section{Resultados}

As concepções que discentes e docentes possuem sobre competências na enfermagem evidenciam diferentes significações, como função própria de um profissional e capacidade para desempenhar uma tarefa com qualidade, envolvendo conhecimento, experiência e valores pessoais. São destacados alguns atributos da competência do profissional enfermeiro e do docente de enfermagem. Estes atributos coincidem com as características do pensador crítico. Além destas, emergiram dos debates nos grupos focais a concepção de dimensões (técnico-científica, humanística, de relacionamento interpessoal, comunicativa, sócio-política, educacional) de competência ${ }^{(9)}$.

Em resposta ao objetivo de relacionar as concepções que os participantes possuem sobre competências na enfermagem, com o processo ensino-aprendizagem do diagnóstico de enfermagem, foi possível apreender elementos explicitados e/ou subjacentes em âmbito relacional, procedimental e contextual. A eles categorizei como Relação com os saberes/conhecimentos, Estratégias de ensinoaprendizagem e Contexto Institucional do Curso.

Os saberes e conhecimentos, termos adotados nesta investigação com sentido similar, longe de serem finalidade última do processo educativo, são recursos que auxiliam o aluno no desenvolvimento da competência. Na Relação com os saberes/conhecimentos acerca do diagnóstico de enfermagem se observa certa ambigüidade dos discentes e docentes, ora posicionando-se contrários ora a favor deste conhecimento.

Os conhecimentos ou saberes consistem em representações que aspiram à verdade, à objetividade e à exatidão. Com algum distanciamento histórico, é possível perceber que os conhecimentos adquiridos pela maioria dos contemporâneos variam entre as sociedades ou em diferentes épocas. São raros os conhecimentos considerados válidos de forma unânime. Assim sendo, em torno de conhecimentos dito fundamentados, existem aqueles controversos, postos em dúvida, em vias de serem retificados ou abandonados; ou conhecimentos emergentes, ainda não confirmados. Uma sociedade é um campo de força onde se defrontam representações contraditórias que almejam ao status de conhecimentos verdadeiros ${ }^{(19)}$. Diante do exposto, é possível considerar o diagnóstico de enfermagem como um exemplo dos conhecimentos controversos, e a sociedade de enfermagem, ou parte dela, luta por seu reconhecimento.

A idéia que muitas vezes perpassa é de que os diagnósticos de enfermagem estão intimamente relacionados à dimensão técnica e o cuidado ao paciente à dimensão humanística, tornando-se opostos e incompatíveis. Isso compreende, na minha opinião, um grande equívoco. O cuidado, para que corresponda às necessidades do paciente, necessita de uma avaliação que abarque a pessoa no seu todo, incluindo aspectos subjetivos e objetivos. Os dados obtidos serão analisados pela enfermeira, que, a partir do seu conhecimento, experiência e intuição, fará um julgamento das respostas apresentadas pelo paciente aos problemas de saúde ou processos de vida, confirmando com ele, sempre que possível, o resultado do seu julgamento de modo a realizar ou participar do cuidado que o paciente requer. Este julgamento é o diagnóstico de enfermagem.

$\mathrm{Na}$ visão inicial dos alunos, para diagnosticar em enfermagem, é suficiente o desenvolvimento da dimensão técnico-científica de competência, aqui considerada no seu direcionamento às necessidades físicas do paciente, associado especialmente às suas necessidades biológicas. Após ampla discussão eles complementaram o seu posicionamento manifestando ser a dimensão humanística que possibilita elaborar diagnósticos de enfermagem individualizados, extrapolando àqueles que são comuns aos pacientes em semelhante situação clínica. Ratificado por docentes e discentes, o Processo de Enfermagem está hoje consolidado como metodologia científica de cuidado ao indivíduo, família e comunidade. O diagnóstico de enfermagem, entretanto, representa uma mudança ainda não digerida por toda a comunidade de enfermagem investigada(20).

Anteriormente à proposta diagnóstica, a segunda etapa do processo de enfermagem contemplava os problemas de enfermagem traduzidos por procedimentos que o paciente realizava, cateteres e sondas que portava, tratamentos medicamentosos a que era submetido, entre outros. Neste prisma, a prescrição de enfermagem era mais direcionada aos cuidados relativos aos procedimentos, aos dispositivos de drenagem e aos medicamentos do que às reações do paciente às situações de saúde/doença ou processos de vida. O diagnóstico de enfermagem impõe mudanças no processo de raciocínio, requerendo competência do docente e do discente.

Nas Estratégias de ensino-aprendizagem foi destacada a importância dos estudos de caso. Esta metodologia, que pode ser entendida como uma situação-problema, é ardorosamente defendida por estudiosos de uma pedagogia diferenciada que visa à construção de competências ${ }^{(8,12,19,21)}$. Sugere-se utilizá-la em situações de complexidade crescente, em que o pensamento crítico e a tomada de decisão sejam incorporados progressivamente. No momento da coleta de dados as dimensões de competência comunicativa e de relacionamento interpessoal, além da técnico-científica, encontram espaço de desenvolvimento, privilegiando, ainda, questões éticas, estéticas, culturais e sociais.

Investir nos processos de pensamento dos alunos, como nas estratégias metacognitivas, indiretamente apontado por uma docente, parece ser um caminho possível e esperado na construção da competência. Elas favorecem a compreensão e o controle dos alunos sobre seus próprios processos de pensamento, como o planejamento, o monitoramento e a avaliação das suas estratégias de solução de problemas $^{(22-25)}$.

Ao final dos quatro encontros os discentes expressaram de forma consensual que o ensino de enfermagem deveria ser fundamentado nos diagnósticos de enfermagem, centralizado primeiramente no processo de raciocínio, embasado nas ciências biológicas, humanas, sociais e da enfermagem. Foi, também, destacada a importância de exercitar a proposta diagnóstica previamente à utilização do sistema informatizado 
do hospital. A acurácia diagnóstica(24) é ainda um objetivo a ser alcançado no ensino e na assistência, já que as diferentes interpretações acerca dos sinais e sintomas do paciente, resultando em diagnósticos de enfermagem distintos, são observadas por discentes e docentes em campo de estágio.

A avaliação da aprendizagem tangenciou os debates de discentes e docentes possivelmente pela falta de conhecimento destes em relação à proposta dos diagnósticos de enfermagem e o desconforto muitas vezes percebido em discutir um tema tratado no ensino e para o qual não se sentem suficientemente preparados. Por vezes, ambos manifestam o quanto o aluno está mais entusiasmado e até melhor preparado que o professor para utilizar os diagnósticos de enfermagem na prática clínica.

Ao avaliar o processo diagnóstico percorrido pelo aluno, observa-se a impossibilidade de o seu ensino ter sido apenas transmissivo, baseado exclusivamente na memorização. Exige, sim, conhecimento abrangente e fundamentado, com certo grau de memorização, mas, acima de tudo, raciocínio, crítica e reflexão. Crítica inclusive acerca dos próprios títulos diagnósticos, muitas vezes estranhos à nossa realidade, ao nosso saber e ao nosso fazer. Críticas que têm sido feitas no interior da própria NANDA, que não pretende ser uma associação engessada, mas receptiva às contribuições dos colegas de diferentes países, de modo a contemplar a diversidade cultural(26).

Quanto ao Contexto Institucional do Curso, foram debatidos aspectos relativos à estrutura curricular e à política institucional da Escola de Enfermagem. A tendência dos discentes é apontar a inadequação curricular direcionada ao ensino dos diagnósticos de enfermagem, atribuída ao pouco aprofundamento e à insuficiência de carga horária para desenvolver conteúdos necessários, especialmente situados na área biológica. Na minha percepção não se trata de aumentar a carga horária das disciplinas básicas, mas de abordar estes conteúdos nas disciplinas de enfermagem, realizando as transversalidades necessárias na determinação de um diagnóstico.

A introdução de inovações exige novas maneiras de planejar, ensinar e avaliar(27). Considero a utilização dos diagnósticos de enfermagem como ponto norteador do ensino nas disciplinas que abordam áreas de interesse da enfermagem e centrado no cuidado ao paciente em lugar da abordagem ainda assumida, cujo foco persiste nos processos patológicos e enfermidades, uma inovação viável e desejável. Não se trata de abdicar dos conhecimentos biológicos e fisiopatológicos, indispensáveis no cuidado ao paciente. Tratase de redirecionar o nosso olhar e o nosso ensino, valorizando essencialmente o conhecimento que é próprio, independente, e que delineia a identidade da profissão.

Foi consenso entre docentes e discentes que a incorporação dos diagnósticos de enfermagem no ensino de graduação precisa ser assumido como política institucional e não somente por iniciativa de professores isolados em algumas disciplinas do currículo. Para os alunos parece evidente a necessidade de adesão dos professores na introdução de uma inovação como a proposta. De acordo com os docentes é preciso decisão institucional quanto ao referencial a ser adotado no ensino, uma vez que o sistema informatizado do hospital universitário contempla os diagnósticos da NANDA conjugados às Necessidades Humanas Básicas ${ }^{(28)}$, diversos da estrutura conceitual dos Padrões de Respostas Humanas utilizada pela própria NANDA em sua Taxonomia $\left.\right|^{(29)}$, ou mesmo na Taxonomia II(30).

Parece-me que distintos sistemas de classificação da prática de enfermagem que vem sendo desenvolvidos em vários países, bem como as diferentes estruturas conceituais empregadas nos diagnósticos da NANDA deveriam ser versadas no ensino, dando-se ênfase ao modelo assumido em nosso hospital universitário onde ocorre grande parte do ensino prático(20).

Esta inovação na metodologia do cuidado e nos registros de enfermagem exige envolvimento e formação continuada dos professores. Implica, como foi dito anteriormente, transição de paradigma, alteração das estratégias curriculares, desalojar convicções, se expor às críticas e observações dos colegas, dos alunos, dos demais profissionais da área da saúde e do indivíduo ou coletividade que requerem nossos cuidados. Exige revisão das próprias resistências e disposição para mudança.

Assumir solidariamente uma mudança, como a utilização dos diagnósticos de enfermagem no currículo do curso em estudo, foi manifestado como desejável. Qualquer modificação importante é uma aposta. Melhor seria assumi-la coletivamente, correndo-se riscos razoáveis de forma solidária. Isso não significa que todos os docentes e lideranças estejam convictos da alteração, bastando uma pequena maioria para levar adiante o sistema, pois inevitavelmente toda mudança divide opiniões. Mas implica aceitar maneiras diferentes ou opostas de pensar ${ }^{(8)}$.

\section{Considerações finais}

Este estudo deu condições de ratificar o pressuposto básico de que o diagnosticar em enfermagem, numa perspectiva integralizadora, abarca as dimensões técnicocientífica, humanística (ética, estética e cultural), de relacionamento pessoal e comunicativa da competência. Evidenciou-se que as habilidades de pensamento crítico e de tomada de decisão, envolvidas na atividade diagnóstica, favorecem a construção da competência do formando em enfermagem. Confirmou-se, também, que o ensinoaprendizagem do diagnóstico de enfermagem requer uma série de competências específicas e habilidades inerentes ao ofício docente e discente.

Os resultados apontaram para a necessidade de formação de professores acerca dos diagnósticos de enfermagem com vistas à construção de competência do discente e do docente, de competência coletiva, com a partilha de experiências e reflexão.

\section{Referências}

1. Horta WA. Processo de enfermagem. São Paulo: EPU; 1979.

2. Garcia TR. O ensino do diagnóstico de enfermagem: a experiência do curso de mestrado em enfermagem na UFPB. In: Anais do IV Simpósio Nacional de Diagnósticos de Enfermagem; 1998 maio 10-43: Curitiba (PR), Brasil. Curitiba (PR): ABEn;1998. p.1-10.

3. Jesus CAC. Raciocínio clínico de graduandos e enfermeiros na construção de diagnósticos de enfermagem [tese de doutorado em Enfermagem]. Ribeirão Preto (SP): Escola de Enfermagem, Universidade de São Paulo; 2000.251f.

4. Cruz DLM. A inserção do diagnóstico de enfermagem no processo assistencial. In: Cianciarullo TI, Gualda DMR, Melleiro MM, Anabuki $\mathrm{MH}$, organizadoras. Sistema de assistência de enfermagem: evolução e tendências. São Paulo: Ícone;2001.p.63-84.

5. Luckesi C. Fazer universidade: uma proposta metodológica. 10a ed. São Paulo: Cortez;1998.

6. Cruz DLM. Contribuições do diagnóstico de enfermagem para a autonomia da enfermeira. In: Guedes MVC, Araújo TL, organizadoras. O uso do diagnóstico na prática da enfermagem. $2^{\mathrm{a}}$ ed. Brasília (DF); ABEn; 1997.p. 51-58.

7. Ministério da Educação (BR), Conselho Nacional de Educação, Câmara de Educação Superior. Resolução $n^{\circ} 3$, de 7 de novembro de 2001: Diretrizes Curriculares Nacionais do Curso de Graduação em Enfermagem. Brasília (DF); 2001. Disponível em: ULR: <http:// www.mec.gov.br/cne/pdf/CES03.pdf>. Acessado em: 25 jun 2004.

8. Perrenoud P. Construir as competências desde a escola. Porto Alegre (RS): Artes Médicas Sul;1999.90p

9. Almeida MA. Concepções de discentes e docentes sobre competência na enfermagem. Revista Gaúcha de Enfermagem, Porto Alegre (RS) 2004 ago;25(2). (No prelo). 
10. Ropé F, Tanguy L, organizadores. Saberes e competências: o uso de tais noções na escola e na empresa. Campinas (SP): Papirus;1997. $207 \mathrm{p}$.

11. Manfredi SM. Trabalho, qualificação e competência profissional: das dimensões conceituais e políticas. Educação e Sociedade, Campinas (SP) 1998 set;19(64):1-20.

12. Perrenoud P. Novas competências para ensinar. Porto Alegre (RS): Artes Médicas Sul;2000.192 p.

13. Rios TA. Compreender e ensinar: por uma docência da melhor qualidade. São Paulo: Cortez; 2001.158 p.

14. Carey MA. The group effect in focus groups: planning, implementing, and interpreting focus group research. In: Morse J. Critical issues in qualitative research methods. Thousand Oaks (CA): Sage Publications;1994.p. 225-41.

15. Dall'agnol C, Trench MH. Grupos focais como estratégia metodológica em pesquisas na enfermagem. Revista Gaúcha de Enfermagem, Porto Alegre (RS) 1999 jan;20(1):5-25.

16. Morrison R, Peoples L. Using focus group methodology in nursing The Journal of Continuing Education in Nursing, Thorofare (NJ) 1999 Mar/Apr;30(2):62-5.

17. Moraes R. Análise de conteúdo. Educação, Porto Alegre (RS) 1999;22(37):7-32.

18. Crossetti MGO, Rodegueri M, DML, Dias VLM. O uso do computador como ferramenta para implementação do processo de enfermagem. Revista Brasileira de Enfermagem, Brasília (DF) 2002 nov/dez; 55(6): 705-8.

19. Perrenoud P. Ensinar: agir na urgência, decidir na incerteza. $2^{\mathrm{a}}$ ed. Porto Alegre: Artmed; 2001.208p.

20. Almeida MA. Sistematização da assistência de enfermagem na formação do enfermeiro. In: Anais do VII Simpósio Nacional de Diagnósticos de Enfermagem; 2004 maio/jun 29-1; Belo Horizonte (MG), Brasil. Belo Horizonte (MG): ABEn; 2004. p.88-97.

21. Rey B. As competências transversais em questão. Porto Alegre (RS): Artmed; 2002. 232p.
22. Antonijevic N, Chadwick C. Estratégias cognitivas e metacognição. Tecnologia Educacional, Rio de Janeiro 1983 mar/abr; 12(51): 20-8.

23. Pesut $D$, Herman J. Metacognitive skills in diagnostic reasoning: making the implicit explicit. Nursing Diagnosis, Philadelphia (PA) 1992 Oct/ Dec;3(4):148-54.

24. Lunney M. Nursing diagnosis, thinking and critical thinking. In: Lunney M. Critical thinking \& nursing diagnosis: case studies \& analyses. Philadelphia (PA): North American Nursing Diagnosis Association; 2001. p. 3-17.

25. Almeida MA. Estratégias metacognitivas: uma possibilidade no ensino de enfermagem. Revista Brasileira de Enfermagem, Brasília (DF) 2002 jul/ago;55(4):424-9.

26. Gordon M. The NANDA Taxonomy II. In: Proceedings of Special Conference of the Association of Common European Nursing Diagnosis, Interventions and Outcome. Viena: Verlag Hans Huber; 2002. p.9-29.

27. Enricone D. O professor e as inovações. In: Enricone D, organizador. Ser professor. Porto Alegre (RS): EDIPUCRS; 2001. p. 41-56.

28. Benedet SA, Bub MBC. Manual de diagnóstico de enfermagem: uma abordagem baseada na teoria das necessidades básicas e na classificação diagnóstica da NANDA. Florianópolis (SC): Bernúcia; 1998.

29. North American Nursing Diagnosis Association. Diagnósticos de enfermagem da NANDA: definições e classificação, 1999-2000. Porto Alegre (RS): Artes Médicas Sul;2000.184p.

30 North American Nursing Diagnosis Association. Diagnósticos de enfermagem da NANDA: definições e classificação, 2001-2002. Porto Alegre (RS): ARTMED;2002.288p.

Data de Recebimento: 13/01/2004

Data de Aprovação: 24/04/2004 